\section{Atoms in strong}

\section{laser fields}

Anne L'Huillier, Lund University, Lund, Sweden

$T$ he field of research "atoms in strong laser fields" born a few years after the invention of the laser in 1960, has evolved considerably during the last two decades owing to the rapid technological development of high-power short-pulse lasers. When a high-power laser is focused into a gas of atoms, the electromagnetic field becomes of the same magnitude as the Coulomb field, which binds a $1 \mathrm{~s}$ electron in a Hydrogen atom $\left(5.1 \times 10^{9} \mathrm{~V} \cdot \mathrm{m}^{-1}\right)$. Three highly nonlinear phenomena, schematically pictured in Figure 1, can happen: Electrons initially in the ground state absorb a large number of photons, many more than the minimum number required for ionisation, thus being ionised with a high kinetic energy. This process, shown for the first time in 1979, is called Above Threshold Ionisation (ATI). Not only one, but many electrons can be emitted from atoms subject to strong laser fields. They can be emitted one at a time, in a sequential process, or simultaneously, a mechanism called direct, or non-sequential. Double ionisation of alkaline earth atoms was observed as early as in 1975 and the first evidence for non-sequential ionisation of rare gas atoms was first demonstrated in 1983. Finally, efficient photon emission in the extreme ultraviolet (XUV) range, in the form of high-order harmonics of the fundamental (linearly-polarised) laser field (HHG), shown for the first time in 1987, can occur.

This field of research remained rather small and exotic through the 1970's and part of the 1980's, restricted, for the experimental part, to a few well-founded research institutes, that could afford expensive laser systems. About fifteen years ago, a new laser material, titanium-sapphire, and a new amplification technique, chirped pulse amplification, made high-power lasers accessible to many university laboratories. This research has become one of the most exciting fields of research in atomic, molecular and optical

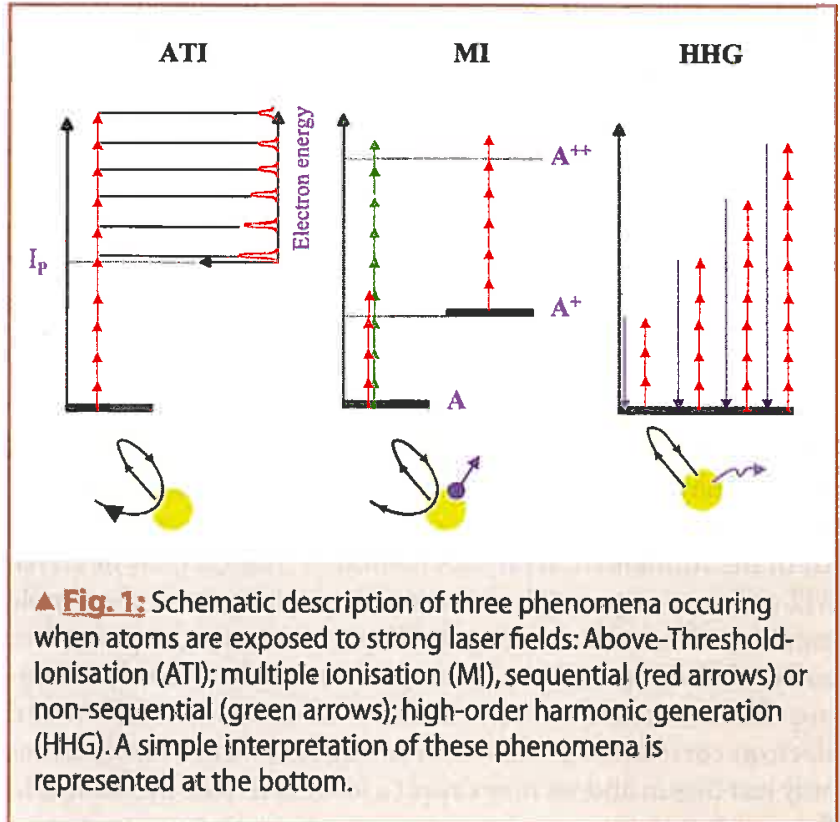

(AMO) physics, attracting a large number of scientists all over the world, with a strong European contribution.

\section{Experiment}

A typical and general experimental setup for studying atoms in strong laser fields is shown in Figure 2. The first element is a short pulse laser. The laser pulses used are often in the femtosecond range, the shortest being sub- $10 \mathrm{fs}$. High repetition rates, today in the $\mathrm{kHz}$ range, allow experimentalists to get good statistics. Focused intensities needed to get into the strong field regime are of the order of $10^{14}-10^{15} \mathrm{~W} \cdot \mathrm{cm}^{-2}$. In the last decade, the favourite tool has become the titanium sapphire laser, operating at $800 \mathrm{~nm}$ in the near-infrared, and providing very short pulse duration, high laser intensity at high repetition rate. A large part of the experimental activity is to make front-line lasers work, and it is no coincidence that many laboratories successful in this field of research also develop these laser systems and their diagnostics. The second part of the experimental setup is a vacuum chamber where a gas of atoms (often rare gases) is being introduced, in a cell or pulsed jet. Ions and/or electrons are detected and analysed using time-of-flight techniques. In addition, angular distribution of the photoelectrons can be recorded. Lately, this field of research has benefited considerably from advances in coincidence and imaging techniques, developed in the AMO physics community working with synchrotron radiation. This has been particularly useful to unravel the mechanism responsible for non-sequential ionisation. In high-order harmonic generation experiments, the atomic density is much higher than in ionisation experiments, up to a few hundreds mbar. The radiation emitted on axis can be detected and analysed using a standard XUV spectrometer, including a grating and a photon detector.

\section{Theory}

The theoretical problem consists in solving the time-dependent Schrödinger equation (TDSE) that describes the interaction of a many-electron atom with a laser field. During many years, theorists have concentrated their effort on solving the problem of a hydrogen atom, or more generally, a single-active electron atom in a strong laser field. A number of methods have been proposed to solve this problem. Two of them stand out: the numerical solution of the TDSE, pioneered by Kulander at the end of the 80 's and the semiclassical strong field approximation (SFA) developed by Lewenstein and others in the 90 's. A large effort is presently being devoted to go beyond the single-active electron approximation. Many insights in the physical understanding of the interaction between atoms and strong laser fields have been provided by a very simple model, called the "simple man's theory", proposed first in 1987 by Van der Linden van der Heuvell and Muller in the context of ATI and later on extended by Corkum and others to the other phenomena shown in Figure 1. According to this model, the electron tunnels through the energy barrier formed by the Coulomb field in the presence of the relatively slowly-varying linearly polarised electric field of the laser. It then undergoes (classical) oscillations in the field, during which the influence of the Coulomb force from the nucleus is practically negligible. If the electron comes back to the vicinity of the nucleus, it may be rescattered one or several times by the nucleus, possibly acquiring a high kinetic energy, and in some cases, kicking out a second or third electron. It may also recombine back to the ground state, thus producing a high energy photon. These effects are illustrated at the bottom of Figure 1. 


\section{Above-threshold-ionisation}

A typical ATI spectrum shows a number of electron peaks separated by the laser photon energy (see Figure 1). For short and intense laser pulses, the ionisation potential $\left(I_{p}\right)$ is increased by the (timedependent) ponderomotive potential, i.e. the mean kinetic energy acquired by an electron oscillating in the laser field $U_{p}=e^{2} E^{2} / 4 \mathrm{mw}^{2}$, where $\mathrm{e}$ is the electron charge, $\mathrm{m}$ is its mass, and $\mathrm{E}$ and $\mathrm{w}$ are the laser electric field and frequency, respectively. Most of the early work, performed in the 1980's, concentrated on the low-energy part of the spectrum, showing, for example, the influence of the ponderomotive potential, the role of AC-Stark shifted resonant excited states and the transition from the multiphoton to the tunneling regime. The experimental precision in detecting electron spectra increased significantly from the mid 90's owing essentially to higher laser repetition rates. Thus ATI spectra with many decades in number of counts could be recorded. Amazingly, the spectra were found to extend over many tens of $\mathrm{eV}$, with a decrease for the first orders, up to $\sim 2 \mathrm{U}_{\mathrm{p}}$, followed by a large plateau extending to $\sim 10 \mathrm{U}_{\mathrm{p}}$. In general, with linear polarisation, electrons are generated along the polarisation's direction. It was found that angular distributions exhibit a much more complex (off-axis) structure at the edge of the plateau, called "scattering rings". The large plateau and the complex angular structure originate from the rescattering of the electron wavepacket on the parent ion.

\section{Multiple ionisation}

The simplest multiple ionisation mechanism for atoms in strong laser fields is the so-called sequential stripping mechanism, i.e. a sequence of single electron ionisation acts: ionisation of the atom, then of the singly charged ion, then of the doubly charged ion and so on. The main experimental effort during the 1980 's was to test the limits of this mechanism with the available laser powers and to understand the process responsible for the ionisation of the different charge states (multiphoton or tunnelling). The existence of a "knee" in the ion signal variation as a function of intensity indicates, however, that sequential ionisation is not the only mechanism responsible for multiple ionisation. A lot of effort has been and is still devoted to the understanding of the nonsequential ionisation process. Several ideas have been proposed, one of them, illustrated in Fig. 1, being electron scattering on a parent ion, leading to ejection of a second electron. Up to a couple of years ago, the experimental method consisted in measuring the number of ions as a function of the laser intensity and in varying the laser pulse characteristics (for example, its polarisation). Progress in experimental techniques with, for example, recoil-ion momentum spectroscopy and electron-ion coincidence measurements allows now scientists to record the energies and angular distributions of the electrons emitted during a multiple ionisation process, thus providing better experimental insight.

\section{High-order harmonic generation}

A high-order harmonic spectrum consists in a sequence of peaks centered at frequency qw, where $q$ is an odd integer. Only odd orders can be observed, owing to inversion symmetry in an atomic gas (In the time domain, this means that the process is periodical with a periodicity twice the laser period). A HHG spectrum has a characteristic behaviour: A fast decrease for the first few harmonics, followed by a long plateau of harmonics with approximately constant intensity. The plateau ends up by a sharp cut-off. Most of the early work on harmonic generation concentrated on the extension of the plateau, i.e. the generation of harmonics of shorter wavelength. Today, harmonic spectra produced with short and intense laser pulses extend to more than 500

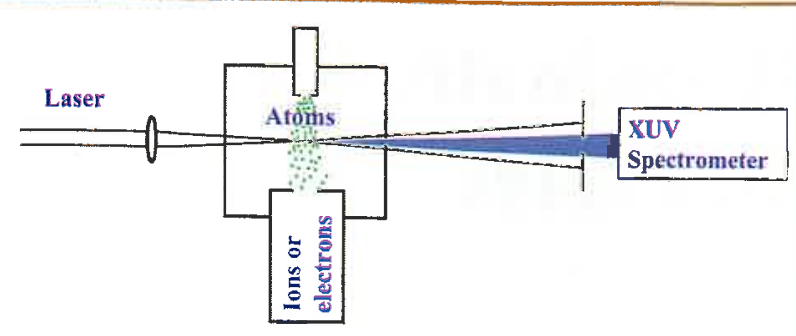

A Fig. 2: Typical schematic experimental setup in the study of atoms in strong laser fields, An intense short pulse laser is focused into an interaction chamber, which contains a gas of atoms. Ions or electrons can be detected, e.g. with time-of-flight techniques. Alternatively, the radiation, which is emitted on axis, can be analysed with an extreme-ultraviolet spectrometer.

$\mathrm{eV}$, down to the water window below the carbon K-edge at $4.4 \mathrm{~nm}$. A large effort has been devoted to optimize and characterize the properties of this new source of XUV radiation. A milestone in the understanding of $H H G$ processes was the finding by Kulander and coworkers in 1992 that the cut-off position in the harmonic spectrum follows the universal law $I_{p}+3 U_{p}$. This result was immediately interpreted in terms of the simple man's theory, and led to the formulation of the SFA. A realistic description of HHG involves, however, not only the calculation of the single atom response, but also the solution of propagation (Maxwell) equations for the emitted radiation.

\section{Attosecond Pulses}

Almost immediately after the first observation of the harmonic plateau at the end of the 1980 's, it was realised that, if the harmonics were emitted in phase, i.e. phase-locked, the temporal structure of the radiation emitted from the medium would consist of a "train" of attosecond pulses separated by half the laser period. There is a clear analogy here with mode-locked lasers, where axial modes oscillating in a laser cavity are locked in phase, leading to the production of trains of short pulses. From a microscopic point of view, at each half-laser cycle, there is a short (attosecond) burst of light, as an electron recombines back to the ground state. Isolated attosecond pulses can be produced if one limits these returns to single events. The simplest idea is to use a very short (7 fs) and intense laser pulse. Such a laser source should allow one to generate single attosecond pulses, because harmonic generation occurs during a limited time interval, before the onset of ionisation. Attosecond pulses have remained, however, essentially a theoretical prediction, until last year. Two important experiments show evidence for trains of 250 as pulses (Agostini, Muller and coworkers), and for isolated 650 as pulses (Krausz and coworkers).

\section{Outlook}

In conclusion, "atoms in strong laser fields" is a fascinating field of AMO physics, that has progressed considerably during the last decade. At the start of the 21st century, we begin to understand a lot of the fundamental physics behind. A unified view of HHG, ATI and non-sequential ionisation, originating from the simple man's model and the strong field approximation, expressed in terms of electron trajectories or quantum paths is slowly emerging. The systematic study of multi-electron ionisation and electron correlation processes in strong laser fields, however, has only just begun and we may expect a lot of activities in this area in the near future. 
This field of research, though quite fundamental, has led to a very interesting application: The harmonic radiation, with its unique properties of ultrashort pulse duration, high brightness and good spatial and temporal coherence, is being used in a growing number of applications ranging from atomic and molecular spectroscopy to solid-state and plasma physics. It has also been proposed as an alternative source for nanolithography, in particular for metrological purposes. It opens up two new fields of research: multiphoton processes in the XUV range, demonstrated for the first time three years ago, and attosecond physics, where processes in atoms and molecules can be studied at an unprecedented time scale. Attophysics is just born and there is already an active discussion on the possible applications of attosecond XUV pulses. The first step towards the use of attosecond pulses has been recently taken by Krausz and his collaborators who have been able to "steer" electron wavepackets, generated by attosecond XUV pulses, in the laser light.

This work has been supported by the Swedish Research Council.

\section{Further Reading}

M. Protopapas, C.H. Keitel, and P.L. Knight, Rep. Prog. Phys. 60, 389

(1997) "Atomic Physics with superhigh intensity lasers".

C. J. Joachain, M. Dörr and N. Kylstra, Adv. Atom. Mol. Opt. Phys. 42, 225 (2000) "High intensity laser-atom physics".

T. Brabec and F. Krausz, Rev. Mod. Phys. 72, 545 (2000). "Intense fewcycle laser fields: Frontiers of nonlinear optics".

M. Lewenstein, Science 297, 1131 (2002) "Resolving atomic process on the attosecond time scale" and references therein. 